\title{
ON A CLASS OF MEROMORPHIC FUNCTIONS
}

\section{N. BAKER}

In his paper [1] F. Gross considers functions $f(z)$ and $g(z)$ meromorphic in the plane and satisfying

$$
f^{n}+g^{n}=1 \text {, }
$$

where $n$ is a fixed integer. For $n=2$ he shows that all meromorphic solutions of (1) are of the form

$$
f=\frac{2 \beta}{1+\beta^{2}}, \quad g=\frac{1-\beta^{2}}{1+\beta^{2}},
$$

where $\beta$ is meromorphic. In this case one may even obtain entire solutions, e.g. $f=\sin z, g=\cos z, \beta=\tan (z / 2)$. Gross also shows that for $n>2$ there are no entire solutions of (1), while for $n>3$ there are no meromorphic solutions.

Now the equation $w^{3}+z^{3}=1$ defines an algebraic function whose Riemann surface has genus 1 , and there is accordingly a uniformization by elliptic functions. If $P(z)$ is the Weierstrass elliptic function with periods $\omega_{1}, \omega_{2}$ satisfying

$$
\left(\rho^{\prime}\right)^{2}=4 \odot^{3}-g_{2} \odot-g_{3}, \quad g_{2}, g_{3} \text { constants, }
$$

then (cf. $\left[2\right.$, p. 227]) $\omega_{1}$ and $\omega_{2}$ may be chosen so that

$$
g_{2}=0, \quad g_{3}=1 \text {. }
$$

With this $\odot(z)$ we find that

$$
\begin{aligned}
& f(z)=\left\{\frac{1}{2}+\frac{\odot^{\prime}(z)}{(12)^{1 / 2}}\right\} / \odot(z), \\
& g(z)=\left\{\frac{1}{2}-\frac{\odot^{\prime}(z)}{(12)^{1 / 2}}\right\} / \odot(z),
\end{aligned}
$$

satisfy

$$
f^{3}+g^{3}=1 \text {. }
$$

The formulas (2) differ from the analogous formulae in [1], which seem to contain an error.

With the aid of the functions in (2) one may verify a conjecture made by F. Gross in [1], viz. that meromorphic solutions of (3) are

Received by the editors March 28, 1966. 
necessarily elliptic functions of entire functions. We shall prove

THEOREM 1. Any functions $F(z), G(z)$, which are meromorphic in the plane and satisfy

$$
F^{3}+G^{3}=1
$$

have the form

$$
F=f(h(z)), \quad G=\eta g(h(z))=\eta f(-h(z))=f\left(-\eta^{2} h(z)\right),
$$

where $f$ and $g$ are the elliptic functions in (2), $h(z)$ is an entire function of $z$ and $\eta$ is a cube-root of unity.

Proof. Write $\rho=\exp (2 \pi i / 3)$. If $F$ and $G$ are meromorphic solutions of (4), then since $F=\left(1-G^{3}\right)^{1 / 3}$ is single-valued, it follows that the multiplicity of any solution $z$ of $G(z)=\rho, \rho^{2}$ or 1 , is a multiple of 3 .

We shall need to discuss the singularities of the inverse function $f_{-1}(w)$ of the $f(z)$ in (2). Since $f(z)$ is a doubly periodic function it has neither finite nor infinite asymptotic values and hence, by Iversen's theorem, all the singularities of $f_{-1}(w)$ are algebraic. We prove that these singularities all lie over $w=\rho, \rho^{2}$ or 1 . First note that $\rho(z)$ has double poles at the points $m \omega_{1}+n \omega_{2}, m, n$ integral, and so $f(z)$ has single poles at these points. $P(z)$ has poles nowhere else, so that the other poles of $f(z)$ are at the zeros of $P(z)$ and hence there are two simple ones in each period parallelogram, since $\odot(z)$ takes each value twice in such a parallelogram, while $P(z)=0$ implies

$$
\left(\odot^{\prime}\right)^{2}=4 \odot^{3}-1=-1 \neq 0 .
$$

Thus altogether $f(z)$ has three simple poles in each period parallelogram $S$, while by differentiation $f^{\prime}(z)$ has three double poles and thus $f$ and $f^{\prime}$ take each value three or six times respectively, in $S$. Now $f^{3}+g^{3}=1$, so that by the first remarks of this proof $f=\rho, \rho^{2}$ and 1 at least triply at each solution. Thus $f$ takes each value $\rho, \rho^{2}$ and 1 precisely at one point in $S$, the derivative $f^{\prime}$ having a double zero at each of these points and at no other points of $S$. Thus all singularities of $f_{-1}(w)$ lie over $w=\rho, \rho^{2}$ and 1 . In particular $w=\infty$ is a regular point of each branch of $f_{-1}(w)$.

We return to the consideration of $F, G$ satisfying (4), and in the neighborhood of any value $z_{0}$, such that $w=F\left(z_{0}\right) \neq \rho, \rho^{2}$, 1 , we take any branch of $f_{-1}(w)$ and form the regular function element

$$
h(z)=f_{-1}(F(z)) .
$$

Now $h(z)$ may be continued analytically along any curve $\gamma$ in the plane without restriction. Obviously the continuation can only fail 
when $\gamma$ reaches a point $z_{1}$ such that $w_{1}=F\left(z_{1}\right)=\rho, \rho^{2}$ or 1 . Denote by $\gamma_{1}$ the arc of $\gamma$ between $z_{0}$ and $z_{1}$, exclusive of the end point $z_{1}$. Then $h(z)$ is regular along $\gamma_{1}$ and for each point $z$ on $\gamma_{1}$ there is a branch of $f_{-1}$ such that $f_{-1}(F(z))=h(z)$. Now $z_{1}$ is a $3 k$-fold solution of $F\left(z_{1}\right)$ $=w_{1}$, so $F(z)=w_{1}+\{\phi(z)\}^{3}$, where $\phi(z)$ is a regular function in the neighborhood $N:\left|z-z_{1}\right|<\delta, \delta>0$, and satisfies $\phi\left(z_{1}\right)=0$. We may suppose $\delta$ chosen so small that for $z$ in $N$ we have $\left|F(z)-w_{1}\right|<1$. Now in the neighborhood $M:\left|w-w_{1}\right|<1$, the only branch points of $f_{-1}(w)$ lie over $w=w_{1}$. For some $z$ in $\gamma_{1} \cap N$ we form $w=F(z)$ in $M$ and choose the branch $f_{-1}(w)$ such that $f_{-1}(F(z))=h(z)$. We note that for neighboring values $z$ we obtain the same branch $f_{-1}(w)$, which indeed has an expansion

$$
f_{-1}(w)=\lambda+P\left(\left(w-w_{1}\right)^{1 / 3}\right), \quad\left|w-w_{1}\right|<(3)^{1 / 2},
$$

where $\lambda$ is a constant and $P(t)$ is a convergent power series in $t$. Thus we must have for all $z$ in $\gamma_{1} \cap N$, using $F=w_{1}+\phi^{3}$, an expression

$$
h(z)=\lambda+P(\mu \phi),
$$

where $\mu$ is a fixed 3rd root of unity and $\phi$ is regular in $N$. This expression gives a regular continuation of $h(z)$ over the value $z_{1}$. Thus we have verified that $h(z)$ can be continued throughout the plane to give (by the monodromy theorem) a function regular in the plane i.e. an entire function.

We now have $F(z)=f(h(z))$ and

$$
\begin{aligned}
F^{3}+G^{3} & =1, \\
f^{3}+g^{3} & =1, \\
f(h)^{3}+g(h)^{3} & =1=F^{3}+g(h)^{3} .
\end{aligned}
$$

Hence $G^{\mathbf{3}}=g(h)^{3}, G=\eta g(h)$, where $\eta$ is (since $G, g(h)$ are regular) a fixed third root of unity. Since $\rho$ is even and $\rho^{\prime}$ is odd we have $f(-z)$ $=g(z)$ and $G$ can also be written $\eta f(-h)$.

We remark finally that (cf. $[2$, p. 168]) $\odot(z)$ has an expansion

$$
\odot(z)=\frac{1}{z^{2}}+\sum_{n=2}^{\infty} C_{n} z^{2 n-2},
$$

where

$$
\begin{gathered}
(n-3)(2 n+1) C_{n}=3\left(C_{2} C_{n-2}+C_{3} C_{n-3}+\cdots+C_{n-2} C_{2}\right), \\
n=4,5,6, \cdots, \\
C_{2}=\frac{1}{20} g_{2}, \quad C_{3}=\frac{1}{28} g_{3} .
\end{gathered}
$$


Since $g_{2}=0, g_{3}=1$, it is easy to prove inductively that $C_{n}=0$ unless $n \equiv 0$ modulo 3. Substitution in (2) shows that $z f(z)$ is a function of $z^{3}$, so that

$$
f(\eta z)=\eta^{2} f(z), \quad \eta^{3}=1 .
$$

This shows that $f\left(-\eta^{2} h(z)\right)=\eta f(-h(z))$, and the proof of the equivalence of the various expressions for $G$ in (5) is complete.

\section{REFERENCES}

1. F. Gross, On the equation $f^{n}+g^{n}=1$, Bull. Amer. Math. Soc. 72 (1966), 86-88.

2. A. Hurwitz and R. Courant, Funktionentheorie, 4 Auflage, Springer, Berlin, 1964.

Imperial College of Science and Technology, London

\section{ON THE BOUNDARY BEHAVIOR OF FUNCTIONS MEROMORPHIC IN THE UNIT DISK}

\section{PETER COLWELL}

1. Introduction. Let $f(z)$ be meromorphic in $D:\{|z|<1\}$, and suppose that the values assumed by $f(z)$ in $D$ lie in a domain $G$ whose boundary $\Gamma$ has positive logarithmic capacity. Then $f(z)$ is of bounded characteristic in $D$ and has finite radial limits $f\left(e^{i \theta}\right)=\lim _{r \rightarrow 1} f\left(r e^{i \theta}\right)$ at almost all points $e^{i \theta}$ on $C:\{|z|=1\}$. (For this and more general theory of meromorphic functions, see [4, pp. $208 \mathrm{ff}$.].) The class of functions satisfying these conditions and having the additional property that $f\left(e^{i \theta}\right)$ belongs to $\Gamma$ almost everywhere on $C$ has been studied by O. Lehto [3] and D. A. Storvick [6], who called it class (L).

If $A$ is a sequence of points in $D$ satisfying $\sum_{a \in A}(1-|a|)<\infty$, the Blaschke product with respect to $A$ in $D$ is the function $B(z ; A)$ $=\prod_{a \in A}[|a|(a-z) / a(1-\bar{a} z)]$. The present note arises from a suggestion by Professor Storvick that the following theorem, established in $[1]$, be extended to functions in class (L). Here we denote by $A^{\prime}$ the derived set of $A$.

Theorem 1. Let $E$ be a set on $C$. A necessary and sufficient condition

Presented to the Society, November 5, 1965; received by the editors February $17,1966$. 